\title{
The 2-adic valuation of plane partitions and totally symmetric plane partitions
}

\author{
William J. Keith \\ Center for Linear Structures and Combinatorics \\ University of Lisbon, Portugal \\ wjk150@cii.fc.ul.pt
}

Submitted: Apr 5, 2011; Accepted: Feb 22, 2012; Published: Feb 29, 2012

\begin{abstract}
This paper confirms a conjecture of Amdeberhan and Moll that the power of 2 dividing the number of plane partitions in an $n$-cube is greater than the power of 2 dividing the number of totally symmetric plane partitions in the same cube when $n$ is even, and less when $n$ is odd.
\end{abstract}

\section{Introduction}

In [1], Tewodros Amdeberhan and Victor H. Moll discussed the 2-adic valuation of the number of alternating sign matrices, plane partitions, and many of the distinguished symmetric subsets of plane partitions, such as totally symmetric self-complementary plane partitions. They conjectured (Conjecture 3.1 in [1]) that

$$
v_{2}\left(P P_{2 n}\right)>v_{2}\left(T S P P_{2 n}\right) \text { and } v_{2}\left(P P_{2 n+1}\right)<v_{2}\left(T S P P_{2 n+1}\right)
$$

where $P P_{n}$ is the number of plane partitions in the $n \times n \times n$ cube, $T S P P_{n}$ is the number of totally symmetric plane partitions in the $n$-cube, i.e. plane partitions symmetric under any permutation of the axes, and $v_{2}(n)$ is the highest power of 2 dividing $n$. The main theorem of this paper is that the conjecture holds.

In the next subsection we introduce all the basic definitions and known properties required for the paper. In Section 2 the conjecture is proved. The positivities of the requisite differences are shown to be implied by the positivity of expressions counting the binary digits in certain intervals of integers, which are then counted and provide the proof. In Section 3 we note that the proof is stronger than necessary, and offer a conjectural refinement: the size of the difference appears to be approximately $5 v_{2}\left(T S S C P P_{2 n}\right)$. Supporting calculations are appended. 


\subsection{Definitions}

A plane partition $\pi$ of an integer $x$ is an array $\pi_{i, j}$ of nonnegative integers in $i, j \geq 1$ such that $\sum \pi_{i, j}=x$, which is nonincreasing in rows and columns, that is, $\pi_{i, j} \geq \pi_{i+1, j}$ and $\pi_{i, j} \geq \pi_{i, j+1}$ for all $i, j$. This condition means that $\pi$ has finite support. The points $\left\{(i, j, k) \mid 0<k \leq \pi_{i, j}\right\}$ constitute the three-dimensional Young diagram of $\pi$. We say $\pi$ is in the $n$-cube if $\pi_{i, j} \leq n$ for all $i, j$ and $\pi_{i, j}>0 \Rightarrow i \leq n$ and $j \leq n$.

Example 1. The following figure illustrates the plane partition

$\begin{array}{llll}1 & 1 & & \\ 4 & 1 & & \\ 4 & 2 & 1 & 1 \\ 5 & 4 & 4 & 1\end{array}$

which exists in the 5-cube, or any larger cube.

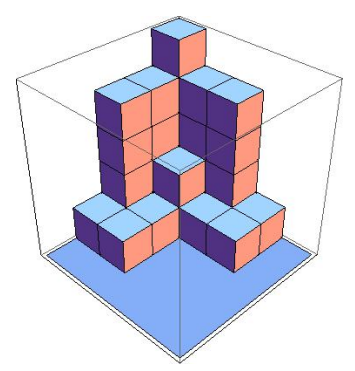

Figure 1: A symmetric plane partition.

We denote the number of plane partitions in the $n$-cube by $P P_{n}$. It has the formula

$$
P P_{n}=\prod_{i, j, k=1}^{n} \frac{i+j+k-1}{i+j+k-2} .
$$

Plane partitions for which the Young diagram is invariant under various involutions of the cube have similarly compact formulas. The set of plane partitions which are invariant under exchange of the $i$ and $j$ axes are called symmetric plane partitions; their number in the $n$-cube is denoted $S P P_{n}$ and has formula

$$
S P P_{n}=\prod_{j=1}^{n} \prod_{i=j}^{n} \frac{i+j+n-1}{i+j-1} .
$$

The plane partitions invariant under any permutations of the three axes are called totally symmetric, and are counted by the formula

$$
T S P P_{n}=\prod_{1 \leq i \leq j \leq k \leq n} \frac{i+j+k-1}{i+j+k-2} .
$$


The plane partition of Example 1 is symmetric but not totally symmetric.

Plane partitions invariant under permutations of the axes, as well as complementation - the operation of taking all lattice points in the $n \times n \times n$ cube not in the Young diagram of the partition, and exchanging corners to make this a new diagram - are called totally symmetric self-complementary partitions. Such partitions only exist in a cube of even size $2 n$. In such a cube, their counting formula is

$$
T S S C P P_{2 n}=\prod_{1 \leq i \leq j \leq n} \frac{i+j+n-1}{i+j+i-1} .
$$

We denote the highest power of 2 dividing $n$ by $v_{2}(n)$, i.e. $v_{2}(n)=k$ if $2^{k} \mid n$ but $2^{k+1} \nmid n$. We use $s_{2}(n)$ to denote the number of $1 \mathrm{~s}$ in the binary expansion of $n$; for instance,

$$
(27)_{2}=11011 \Rightarrow s_{2}(27)=4 \text {. }
$$

This function has the properties that

$$
s_{2}(2 j+1)=1+s_{2}(j), \quad s_{2}(2 j)=s_{2}(j), \quad \text { and } \quad s_{2}(3 m+1) \leq 2 s_{2}(m)+1 .
$$

These two quantities are related by the formula $v_{2}(m !)=m-s_{2}(m)$, due to Legendre, which will repeatedly be useful in the following.

\section{Proof of the Main Result}

Begin by noting that every triple $(i, j, k)$ which indexes a factor in the formula for $T S P P_{n}$ also indexes a factor in the fomula for $P P_{n}$, and the factors so indexed are the same. Triples $(i, j, k)$ from $P P_{n}$ do not index factors in $T S P P_{n}$ if $i>j$, regardless of the relation of $k$ to $i$ and $j$, or if $i \leq j$ but $j>k$, whether $i>k$ or not.

Thus:

$$
P P_{n}=T S P P_{n} \cdot \prod_{j=1}^{n} \prod_{i=j+1}^{n} \prod_{k=1}^{n} \frac{i+j+k-1}{i+j+k-2} \cdot \prod_{i=1}^{n} \prod_{j=i}^{n} \prod_{k=1}^{j-1} \frac{i+j+k-1}{i+j+k-2} .
$$

With the two values of interest isolated, the proof of the conjecture is reduced to determining whether the 2-adic valuation of the outstanding products are positive or negative.

Many of the factors in the numerator and denominator of each product cancel. In the middle term of $(1)$,

$$
\prod_{j=1}^{n} \prod_{i=j+1}^{n} \prod_{k=1}^{n} \frac{i+j+k-1}{i+j+k-2}
$$

most factors arising from a triple $(i, j, k)$ in the numerator cancel with the factor arising from $(i, j, k+1)$ in the denominator. The exceptions are triples with $k=n$ in the numerator, and those with $k=1$ in the denominator. Thus, this term becomes

$$
\prod_{i=1}^{n} \prod_{j=i+1}^{n} \frac{i+j+n-1}{i+j-1}=S P P_{n} \cdot \prod_{i=1}^{n} \frac{2 i-1}{2 i+n-1} \text {. }
$$


That is, this term is exactly the formula for $S P P_{n}$ except that it is missing the boundary where $i=j$.

The 2 -adic valuation of the numerator on the right-hand side is 0 , since $v_{2}(2 i-1)=0$. If $n$ is even, each $2 i+n-1$ is odd, and so the 2 -adic valuation of the denominator is also 0 . If $n$ is odd, $v_{2}(2 i+n-1)>0$, and we must determine its contribution. Over the whole product, we can add in intervening odd factors to create a factorial without changing the 2 -adic valuation of the product, to get

$$
\begin{aligned}
v_{2}\left(\prod_{i=1}^{n} 2 i+n-1\right) & =v_{2}((3 n-1) !)-v_{2}((n-1) !) \\
& =3 n-1-s_{2}(3 n-1)-(n-1)+s_{2}(n-1) \\
& =2 n-s_{2}(3 n-1)+s_{2}(n-1) .
\end{aligned}
$$

In the third factor of (1),

$$
\prod_{i=1}^{n} \prod_{j=i}^{n} \prod_{k=1}^{j-1} \frac{i+j+k-1}{i+j+k-2}
$$

the factor indexed by a triple $(i, j, k)$ in the numerator cancels with the factor indexed by $(i+1, j, k)$ in the denominator unless $i=j$ in the numerator (if $i=n, i=j$ automatically), or $i=1$ in the denominator. We are left with

$$
\prod_{i=1}^{n} \prod_{j=i}^{n} \prod_{k=1}^{j-1} \frac{i+j+k-1}{i+j+k-2}=\prod_{j=1}^{n} \prod_{k=1}^{j-1} \frac{2 j+k-1}{j+k-1}=\prod_{j=1}^{n} \frac{(2 j)(2 j+1) \cdots(3 j-2)}{j(j+1) \cdots(2 j-2)} .
$$

The 2-adic valuation of this product is

$$
\begin{aligned}
& v_{2}\left(\prod_{j=1}^{n} \frac{(2 j)(2 j+1) \cdots(3 j-2)}{j(j+1) \cdots(2 j-2)}\right) \\
&=\sum_{j=1}^{n} v_{2}((3 j-2) !)-v_{2}((2 j-1) !)-v_{2}((2 j-2) !)+v_{2}((j-1) !) \\
&=\sum_{j=1}^{n} 3 j-2-s_{2}(3 j-2)-(2 j-1)+s_{2}(2 j-1) \\
& \quad-(2 j-2)+s_{2}(2 j-2)+j-1-s_{2}(j-1) \\
&=\sum_{j=1}^{n} s_{2}(2 j-1)+s_{2}(2 j-2)-s_{2}(3 j-2)-s_{2}(j-1) \\
&=\sum_{j=1}^{n} s_{2}(j-1)+1+s_{2}(j-1)-s_{2}(3 j-2)-s_{2}(j-1) \\
&= \sum_{j=1}^{n} s_{2}(j-1)+1-s_{2}(3 j-2)=n+\sum_{j=1}^{n} s_{2}(j-1)-s_{2}(3 j-2) .
\end{aligned}
$$


Finally, we determine $v_{2}\left(S P P_{n}\right)$ :

$$
\begin{aligned}
v_{2}\left(S P P_{n}\right)= & v_{2}\left(\prod_{j=1}^{n} \frac{(2 j+n-1)(2 j+n) \cdots(j+2 n-1)}{(2 j-1)(2 j) \cdots(j+n-1)}\right) \\
= & v_{2}\left(\frac{(3 n-1)}{(2 n-1)} \cdot \frac{(3 n-2)(3 n-3)}{(2 n-2)(2 n-3)} \cdot \frac{(3 n-3)(3 n-4)(3 n-5)}{(2 n-3)(2 n-4)(2 n-5)} \times\right. \\
& \left.\cdots \times \frac{(2 n)(2 n-1) \cdots(n+1)}{(n)(n-1) \cdots(1)}\right) \\
= & v_{2}\left(\frac{(3 n-1) !}{(2 n-1) !} \cdot \frac{(3 n-3) !}{(2 n-2) !} \cdot \frac{(3 n-5) !}{(2 n-3) !} \cdots \frac{(n+1) !}{n !}\right) \\
& /\left(\frac{(2 n-1) !}{(n-1) !} \cdot \frac{(2 n-3) !}{(n-2) !} \cdots \frac{1 !}{0 !}\right) \\
= & \sum_{j=1}^{n} 3 n+1-2 j-s_{2}(3 n+1-2 j)+n-j-s_{2}(n-j) \\
& -(2 n-j)+s_{2}(2 n-j)-(2 n+1-2 j)+s_{2}(2 n+1-2 j) \\
= & \sum_{j=1}^{n} s_{2}(2 n-j)+s_{2}(2 n+1-2 j)-s_{2}(3 n+1-2 j)-s_{2}(n-j) \\
= & n+\sum_{j=1}^{n} s_{2}(2 n-j)-s_{2}(3 n+1-2 j) .
\end{aligned}
$$

Collecting the calculations so far, we have: if $n$ is even,

$$
v_{2}\left(P P_{n}\right)=v_{2}\left(T S P P_{n}\right)+2 n+\sum_{j=1}^{n} s_{2}(2 n-j)-s_{2}(3 n+1-2 j)+s_{2}(j-1)-s_{2}(3 j-2)
$$

and if $n$ is odd,

$$
\begin{aligned}
v_{2}\left(P P_{n}\right)= & v_{2}\left(T S P P_{n}\right)+s_{2}(3 n-1)-s_{2}(n-1) \\
& +\sum_{j=1}^{n} s_{2}(2 n-j)-s_{2}(3 n+1-2 j)+s_{2}(j-1)-s_{2}(3 j-2) .
\end{aligned}
$$

We now examine the two cases separately.

Case 1: Even. The even-subscript part of the conjecture is implied by showing that for any $n$ the 2-adic valuation of the factors other than $v_{2}\left(T S P P_{2 n}\right)$ on the right-hand side of (1) is positive:

$$
\begin{aligned}
v_{2}\left(\prod_{i=1}^{n} \prod_{j=i+1}^{n}\right. & \left.\prod_{k=1}^{n} \frac{i+j+k-1}{i+j+k-2} \cdot \prod_{i=1}^{n} \prod_{j=i}^{n} \prod_{k=1}^{j-1} \frac{i+j+k-1}{i+j+k-2}\right) \\
& =2 n+\sum_{j=1}^{n} s_{2}(2 n-j)+s_{2}(j-1)-s_{2}(3 j-2)-s_{2}(3 n+1-2 j)>0 .
\end{aligned}
$$


We will prove a slightly stronger statement, by subtracting a nonnegative value from the left and middle terms of inequality (2): the 2-adic valuation of $T S S C P P_{2 n}$, the factors for which are a subset of the factors in the expression. The resulting expression in the middle term will be simpler and still strictly positive.

$$
\begin{aligned}
\text { TSSCPP } & =\prod_{1 \leq i \leq j \leq n} \frac{i+j+n-1}{i+j+i-1} \\
= & \frac{(3 n-1)}{(3 n-1)} \cdot \frac{(3 n-3)(3 n-2)}{(3 n-4)(3 n-3)} \cdot \frac{(3 n-5)(3 n-4)(3 n-3)}{(3 n-7)(3 n-6)(3 n-5)} \cdots \frac{(n+1)(n+2) \cdots(2 n)}{(2)(3) \cdots(n+1)} \\
= & {\left[\frac{(3 n-1) !}{(2 n-1) !} \cdot \frac{(3 n-3) !}{(2 n-2) !} \cdot \frac{(3 n-5) !}{(2 n-3) !} \cdots \frac{(n+1) !}{(n) !}\right] } \\
& /\left[\frac{(3 n-1) !}{(3 n-2) !} \cdot \frac{(3 n-3) !}{(3 n-5) !} \cdot \frac{(3 n-5) !}{(3 n-8) !} \cdots \frac{(n+1) !}{(1) !}\right]
\end{aligned}
$$

Thus

$$
\begin{aligned}
v_{2}\left(\text { TSSCPP }_{2 n}\right) & =v_{2}\left(\frac{(3 n-2) !(3 n-5) ! \cdots(1) !}{(2 n-1) !(2 n-2) ! \cdots(n) !}\right) \\
& =\sum_{j=1}^{n} 3 j-2-s_{2}(3 j-2)-(j+n-1)+s_{2}(j+n-1) \\
& =\sum_{j=1}^{n} 2 j-1-n-s_{2}(3 j-2)+s_{2}(j+n-1) \\
& =\sum_{j=1}^{n} s_{2}(j+n-1)-s_{2}(3 j-2) .
\end{aligned}
$$

Subtracting this from the left-hand side of inequality (2), we get

$$
\begin{aligned}
2 n+ & \sum_{j=1}^{n} s_{2}(2 n-j)-s_{2}(3 n+1-2 j)+s_{2}(j-1)-s_{2}(3 j-2)-s_{2}(j+n-1)+s_{2}(3 j-2) \\
& =2 n+\sum_{j=1}^{n} s_{2}(j-1)-s_{2}(3 n+1-2 j)=n+\sum_{j=1}^{n} s_{2}(j-1)-s_{2}((3 n / 2)-j) \\
& =n+\sum_{j=1}^{n} s_{2}(j-1)-s_{2}(j-1+(n / 2))=n+\sum_{j=0}^{n-1} s_{2}(j)-s_{2}(j+(n / 2)) \\
& =2 k+\sum_{j=0}^{2 k-1} s_{2}(j)-s_{2}(j+k)=2 k+\sum_{j=0}^{k-1} s_{2}(j)-\sum_{j=0}^{k-1} s_{2}(j+2 k)
\end{aligned}
$$

where we have set $k=\frac{n}{2}$.

Let $S_{E}(k)=2 k+\sum_{j=0}^{k-1} s_{2}(j)-\sum_{j=0}^{k-1} s_{2}(j+2 k)$. We will now show that this expression is strictly positive. Expressed in terms of the digits being counted, this is the claim that 
if the total number of binary digits for all numbers from 0 to $k-1$ is compared to that for the interval of the same length moved up $2 k$, the excess of binary digits in the latter over the former is no more than $2 k$.

Lemma 1. For $k>0$, we have $\sum_{j=0}^{k-1} s_{2}(j+2 k)-\sum_{j=0}^{k-1} s_{2}(j)<2 k$.

Proof: Let $k=2^{i_{1}}+\cdots+2^{i_{r}}, i_{1}<\cdots<i_{r}$. List the binary expansions of the numbers in the intervals of interest, and label columns by the associated power of 2 , from 0 through $i_{r}+2$. We will concern ourselves with three contributions: $1 \mathrm{~s}$ in the binary expansion that appear in columns $i_{r}+1$ and $i_{r}+2$, the difference in the number of 1 s appearing within the interval in column $i_{r}$, and the differences in the number of 1 s appearing in all columns below $i_{r}$.

\begin{tabular}{|c|c|c|c|c|c|c|c|c|c|c|c|c|c|}
\hline & 0 & 1 & 2 & 3 & 4 & 5 & & 0 & 1 & 2 & 3 & 4 & 5 \\
\hline 0 & 0 & 0 & 0 & 0 & 0 & 0 & 22 & 0 & 1 & 1 & 0 & 1 & 0 \\
\hline 1 & 1 & 0 & 0 & 0 & 0 & 0 & 23 & 1 & 1 & 1 & 0 & 1 & 0 \\
\hline 2 & 0 & 1 & 0 & 0 & 0 & 0 & 24 & 0 & 0 & 0 & 1 & 1 & 0 \\
\hline 3 & 1 & 1 & 0 & 0 & 0 & 0 & 25 & 1 & 0 & 0 & 1 & 1 & 0 \\
\hline 4 & 0 & 0 & 1 & 0 & 0 & 0 & 26 & 0 & 1 & 0 & 1 & 1 & 0 \\
\hline 5 & 1 & 0 & 1 & 0 & 0 & 0 & 27 & 1 & 1 & 0 & 1 & 1 & 0 \\
\hline 6 & 0 & 1 & 1 & 0 & 0 & 0 & 28 & 0 & 0 & 1 & 1 & 1 & 0 \\
\hline 7 & 1 & 1 & 1 & 0 & 0 & 0 & 29 & 1 & 0 & 1 & 1 & 1 & 0 \\
\hline 8 & 0 & 0 & 0 & 1 & 0 & 0 & 30 & 0 & 1 & 1 & 1 & 1 & 0 \\
\hline 9 & 1 & 0 & 0 & 1 & 0 & 0 & 31 & 1 & 1 & 1 & 1 & 1 & 0 \\
\hline 10 & 0 & 1 & 0 & 1 & 0 & 0 & 32 & 0 & 0 & 0 & 0 & 0 & 1 \\
\hline
\end{tabular}

Figure 2: The intervals of interest for $k=11$.

Consider the columns $i_{r}+1$ and $i_{r}+2$, which have no 1 s for the interval $[0, k-1]$. In the interval $[2 k, 3 k-1]$, the smallest value, $2 k$, has exactly one 1 in column $i_{r}+1$, but no $1 \mathrm{~s}$ in any higher column. It is never the case that columns $i_{r}+1$ and $i_{r}+2$ both contain a 1 for numbers in $[2 k, 3 k-1]$, since $2^{i_{r}+1}+2^{i_{r}+2} \geq 3 k+3$. Hence columns $i_{r}+1$ and $i_{r}+2$ (and beyond) together contain exactly one 1 for each number in the interval $[2 k, 3 k-1]$, $k$ more than appear in the corresponding positions for the interval $[0, k-1]$. Thus we must show that fewer than $k$ additional 1 s are added in the columns 0 through $i_{r}$.

The digits in column $i$ have period $2^{i+1}$, consisting of a string of $2^{i} 0 \mathrm{~s}$ followed by $2^{i}$ 1s. If $k=2^{i_{r}}$, then the interval $[0, k-1]=\left[0,2^{i_{r}}-1\right]$ consists of even multiples of the periods for columns 0 through $i_{r}-1$, and column $i_{r}$ consists entirely of 0 s. The interval $[2 k, 3 k-1]=\left[2^{i_{r}+1}, 2^{i_{r}+1}+2^{i_{r}}-1\right]$ will have exactly the same entries in columns 0 through $i_{r}$.

When $k>2^{i_{r}}$, column $i_{r}$ consists of a string of 0 s followed by a string of $1 \mathrm{~s}$ of length $2^{i_{1}}+\cdots+2^{i_{r-1}}$. Let $k^{-}=2^{i_{1}}+\cdots+2^{i_{r-1}} \leq 2^{i_{r}}-1$. The digits in column $i_{r}$ will be rotated forward by $2 k^{-}$, since adding $2 \cdot 2^{i_{r}}$ only moves the frame forward an even period in column $i_{r}$. The difference $a d d\left(i_{r}\right)$ between the number of 1 s in the resulting column, 
and those that existed in the original, will be a fraction of $2^{i_{r}}$ given by a piecewise linear function of the fraction of $2^{i_{r}}$ represented by $k^{-}$.

If $k^{-}$is less $\frac{1}{3} 2^{i_{r}}$, rotating the column upward $2 k^{-}$only brings new $1 \mathrm{~s}$, a total of $2 k^{-}$ of them. If $k^{-}$is at least $\frac{1}{3} 2^{i_{r}}$ but at most $\frac{1}{2} 2^{i_{r}}$, all $2^{i_{r}} 1 \mathrm{~s}$ appear. As $k^{-}$grows from $\frac{1}{2} 2^{i_{r}}$ to $\frac{1}{3} 2^{i_{r}}$, the lowest 1 s rotate out of the interval, until $k^{-}$is $\frac{2}{3} 2^{i_{r}}$ or larger, at which point both the lower and the upper interval both contain the entire string of 0s and the same number of $1 \mathrm{~s}$ - at the top in the case of the lower interval, and partially above and below in the case of the upper.

$$
\frac{a d d\left(i_{r}\right)}{2^{i_{r}}}=\left\{\begin{array}{cc}
2\left(\frac{k^{-}}{2^{i_{r}}}\right) & \left(\frac{k^{-}}{2^{i_{r}}}\right)<\frac{1}{3} \\
1-\left(\frac{k^{-}}{2^{i_{r}}}\right) & \frac{1}{3}<\left(\frac{k^{-}}{2^{i_{r}}}\right) \leq \frac{1}{2} \\
2-3\left(\frac{k^{-}}{2^{i_{r}}}\right) & \frac{1}{2}<\left(\frac{k^{-}}{2^{i_{r}}}\right)<\frac{2}{3} \\
0 & \frac{2}{3}<\left(\frac{k^{-}}{2^{i_{r}}}\right)<1
\end{array}\right.
$$

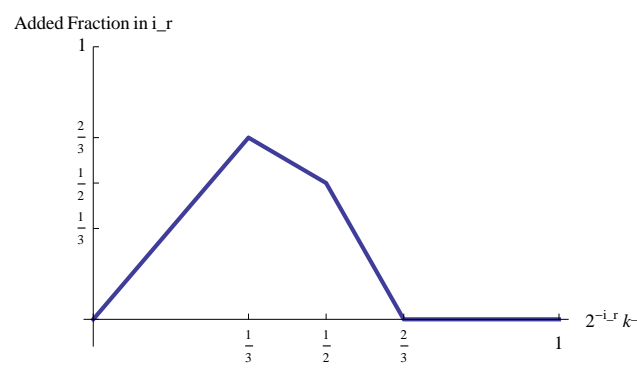

Figure 3: The function $\operatorname{add}\left(i_{r}\right)$.

Therefore, at most, the additional 1 s in column $i_{r}$ contribute $\frac{2}{3} 2^{i_{r}}$ to the difference in the digit sums. But having the digit 1 in column $i_{r}$ contributes $2^{i_{r}}$ to the value of $k$. If we are attempting to add $k 1 \mathrm{~s}$, this leaves a deficit of at least $\frac{1}{3} 2^{i_{r}}$ which, we will see, cannot be made up in lower columns.

In lower columns, we perform an analysis similar to the above, with the additional consideration that the column within the frame of interest may or may not end with a 1. Therefore, in looking at column $\mathrm{b}$, whether $2^{b}$ is a digit in $k$ will also concern us. In general, let $k_{b}=\sum_{i_{j} \leq b} 2^{i_{j}}$. Then the additional number of $1 \mathrm{~s}$ in column $b$ is given by the piecewise linear function

$$
\frac{a d d(b)}{2^{b}}=\left\{\begin{array}{cl}
0 & 0 \leq\left(\frac{k_{b}}{2^{b+1}}\right)<\frac{1}{6} \\
2\left(\frac{k_{b}}{2^{b+1}}\right)-\frac{1}{3} & \frac{1}{6} \leq\left(\frac{k_{b}}{2^{b+1}}\right)<\frac{1}{3} \\
1-2\left(\frac{k_{b}}{2^{b+1}}\right) & \frac{1}{3} \leq\left(\frac{k_{b}}{2^{b+1}}\right)<\frac{1}{2} \\
-2+4\left(\frac{k_{b}}{2^{b+1}}\right) & \frac{1}{2} \leq\left(\frac{k_{b}}{2^{b+1}}\right)<\frac{2}{3} \\
\frac{10}{3}-4\left(\frac{k_{b}}{2^{b+1}}\right) & \frac{2}{3} \leq\left(\frac{k_{b}}{2^{b+1}}\right)<\frac{5}{6} \\
0 & \frac{5}{6} \leq\left(\frac{k_{b}}{2^{b+1}}\right)<1
\end{array}\right.
$$




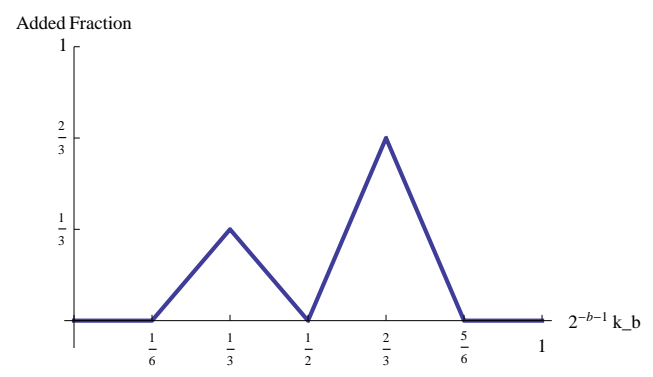

Figure 4: The function $a d d(b)$ for $b<i_{r}$.

Anywhere $\frac{k_{b}}{2^{b+1}} \geq \frac{1}{2}$, the digit in $k$ in column $b$ must be a 1 , contributing $2^{b}$ to the value of $k$. In such a case, the extra 1s number less than the addition to the value of $k$, and so the deficit between $k$ and the number of added 1s increases. If $\frac{k_{b}}{2^{b+1}}<\frac{1}{2}$, the most that can be added is $\frac{1}{3} 2^{b}$. Supposing this to be the case for as many $b$ as possible, the sum still must be at most $\frac{1}{3}\left(1+2+\cdots+2^{i_{r}-1}\right)<\frac{1}{3} 2^{i_{r}}$. Thus each column either increases the deficit, or is insufficient a contribution to close the gap.

Hence fewer than $k 1$ s are added in the columns up to and including $i_{r}$, and exactly $k 1$ s are added in the columns beyond, so the additional 1 s number less than $2 k$. The lemma, and thus this case of the theorem, is proved.

Case 2: Odd. For $n$ odd,

$$
\begin{aligned}
& \sum_{j=1}^{n} s_{2}(2 n-j)-s_{2}(3 n+1-2 j)+s_{2}(j-1)-s_{2}(3 j-2)-v_{2}\left(T S S C P P_{2 n}\right) \\
&= \sum_{j=1}^{n} s_{2}(2 n-j)-s_{2}(3 n+1-2 j)+s_{2}(j-1)-s_{2}(3 j-2) \\
&-\left(\sum_{j=1}^{n} s_{2}(j+n-1)-s_{2}(3 j-2)\right) \\
&= \sum_{j=1}^{n} s_{2}(j-1)-s_{2}(3 n+1-2 j)=\sum_{j=0}^{n-1} s_{2}(j)-s_{2}\left(\frac{n+1}{2}+j\right) \\
&= \sum_{j=0}^{\frac{n-1}{2}} s_{2}(j)-s_{2}(n+j)=\sum_{j=0}^{k-1} s_{2}(j)-s_{2}(j+2 k+1) \\
&= s_{2}(n)-s_{2}(3(n-1) / 2+1)+\sum_{j=0}^{k-1} s_{2}(j)-s_{2}(j+2 k) \\
&= 1+s_{2}(n-1)-s_{2}(3 n-1)+\sum_{j=0}^{k-1} s_{2}(j)-s_{2}(j+2 k)
\end{aligned}
$$


where we now set $k=\frac{n-1}{2}$. Hence

$$
\begin{array}{r}
s_{2}(3 n-1)-s_{2}(n-1)+\sum_{j=1}^{n} s_{2}(2 n-j)-s_{2}(3 n+1-2 j)+s_{2}(j-1)-s_{2}(3 j-2) \\
=v_{2}\left(T S C P P_{2 n}\right)+1+\sum_{j=0}^{k-1} s_{2}(j)-s_{2}(j+2 k) .
\end{array}
$$

By the arguments in the previous section, $\sum_{j=0}^{k-1} s_{2}(j)-s_{2}(j+2 k)$ is negative, and at least $k$. But $v_{2}\left(T S C P P_{2 n}\right)$ is known $([2])$ to be much less than $\frac{n-1}{2}$ :

$$
\max v_{2}\left(T S S C P P_{2 n}\right)=\left[\frac{2^{j}}{3}\right] \quad \text { for } \quad 2 n \in\left[\frac{2}{3} 2^{j}, \frac{4}{3} 2^{j}\right]
$$

where $[x]$ is the closest integer to $x$, and $v_{2}\left(T S S C P P_{2 n}\right)$ is less than half this maximum value on this interval outside its middle portion, $\left[\frac{5}{6} 2^{n}, \frac{7}{6} 2^{n}\right]$.

Thus the right-hand side of (3) is always negative, and so the conjecture is a theorem.

\section{Relative Sizes}

Having shown the sign of the difference between $v_{2}\left(P P_{n}\right)$ and $v_{2}\left(T S P P_{n}\right)$, a natural question is to ask about the size of the difference.

For even index, the difference $v_{2}\left(P P_{2 n}\right)-v_{2}\left(T S P P_{2 n}\right)$ appears to be very nearly 5 times the value $v_{2}\left(T S S C P P_{2 n}\right)$. Below is the graph of the values $v_{2}\left(P P_{2 n}\right)-v_{2}\left(T S P P_{2 n}\right)$ and $v_{2}\left(T S S C P P_{2 n}\right)$.

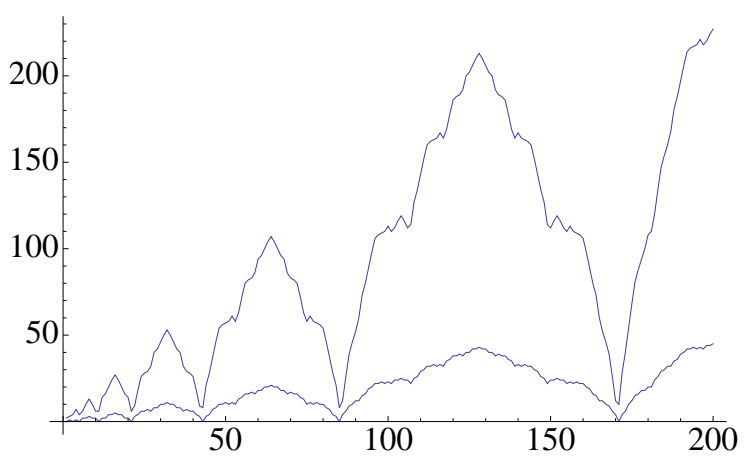

Figure 5: $v_{2}\left(P P_{2 n}\right)-v_{2}\left(T S P P_{2 n}\right)$ and $v_{2}\left(T S S C P P_{2 n}\right)$.

Next we display the ratio $\frac{5 v_{2}\left(T S S C P P_{2 n}\right)}{v_{2}\left(P P_{2 n}\right)-v_{2}\left(T S P P_{2 n}\right)}$. The ratio is eccentric near the very low values of $v_{2}\left(P P_{2 n}\right)-v_{2}\left(T S P P_{2 n}\right)$ (these occur at the Jacobsthal numbers), but for the 


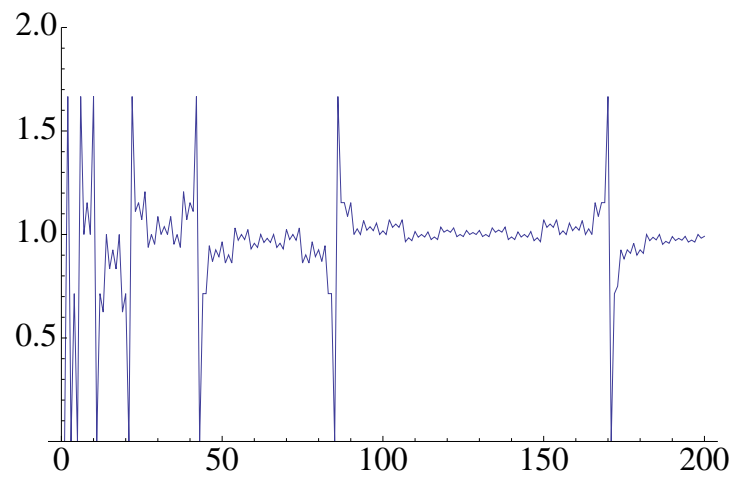

Figure 6: $\left(5 v_{2}\left(T S S C P P_{2 n}\right)\right) /\left(v_{2}\left(P P_{2 n}\right)-v_{2}\left(T S P P_{2 n}\right)\right)$.

most part of the interval is very close to 1 . The maximum ratios appear to be exactly $5 / 3$.

For odd index, the difference $v_{2}\left(P P_{2 n-1}\right)-v_{2}\left(T S P P_{2 n-1}\right)$ decreases steadily, its minima lying close to the line $-2 n+1$. Figure 7 displays these two values.

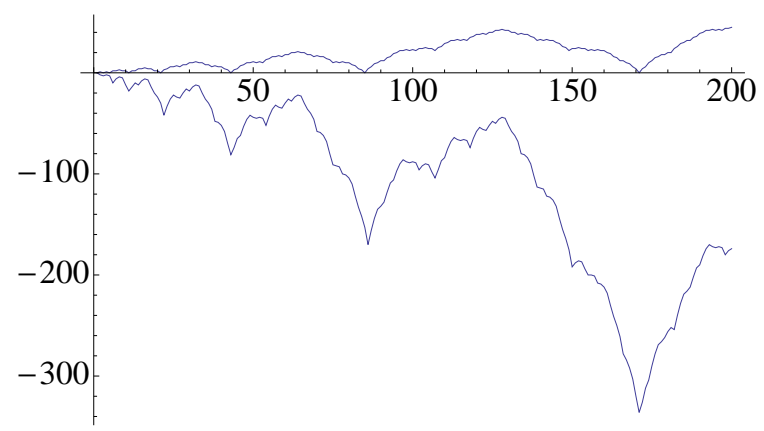

Figure 7: $v_{2}\left(P P_{2 n-1}\right)-v_{2}\left(T S P P_{2 n-1}\right)$.

Finally, Figure 8 gives the ratio $\frac{5 v_{2}\left(T S S C P P_{2 n}\right)}{v_{2}\left(P P_{2 n-1}\right)-v_{2}\left(T S P P_{2 n-1}\right)+2 n}$. The maxima of this ratio are not constant, but again for most $n$ the ratio appears to be close to exactly 1 .

(For interested readers, symbolic computation packages such as Mathematica can generate these values over much larger ranges in just a few seconds, using their built-in algorithms for binary digit conversion and list counting, and the formulas developed in this paper.)

Combining the two cases and noting that extreme values in both cases occur near the Jacobsthal numbers, we can formalize the conjectures thus:

Conjecture 1. For any $\epsilon$, the arithmetic density of whole numbers $n$ on which

$$
\left|1-\frac{5 v_{2}\left(T S S C P P_{2 n}\right)}{v_{2}\left(P P_{2 n}\right)-v_{2}\left(T S P P_{2 n}\right)}\right|<\epsilon
$$




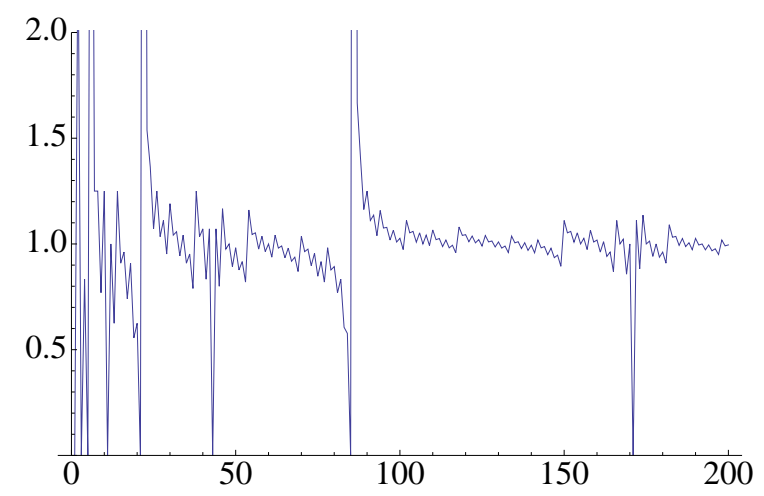

Figure 8: $\left(5 v_{2}\left(T S S C P P_{2 n}\right)\right) /\left(v_{2}\left(P P_{2 n-1}\right)-v_{2}\left(T S P P_{2 n-1}\right)+2 n\right)$.

and

$$
\left|1-\frac{5 v_{2}\left(T S S C P P_{2 n}\right)}{v_{2}\left(P P_{2 n-1}\right)-v_{2}\left(T S P P_{2 n-1}\right)+2 n}\right|<\epsilon
$$

approaches 1 as $n \rightarrow \infty$, with failing values all lying in neighborhoods of Jacobsthal numbers of sizes depending on $\epsilon$.

It seems likely that the proof will employ the fact that the ordered triples comprising $T S P P_{n}$ are just $1 / 6$ the cube of triples comprising $P P_{n}$ in their respective formulae. However, we offer the conjecture to the reader in its present form.

\section{References}

[1] T. Amdeberhan and V. H. Moll, Arithmetic properties of plane partitions, Electron. J. Combin. 18(2), 2011, \#P1.

[2] X. Sun and V. H. Moll, The $p$-adic valuation of sequences counting alternating sign matrices, J. Integer Seq. 12(3), 2009, Article 09.3.8. 